\title{
Three Dimensional Imaging for Drug Discovery
}

\author{
Michail A. Esterman* \\ *Lilly Research Labs, Inc Lilly Corporate Center, Indianapolis, IN 46285
}

The value of direct observation has been valuable from early history, man's curiosity and desire to study the world, observation and measurement of object properties have been fundamental principles [1]. The development of imaging tools such as the microscope, extended the range of natural vision and enabled scientist to observe, record and measure otherwise inaccessible object properties by means of their images. Digital, computer aided microscopy and image analysis further extend unbiased observation. The introduction of commercial Confocal Laser Scanning Microscopes in the 1980's provided scientists with the ability to optically section fluorescence labeled specimens and reconstruct two dimensional and three dimensional views. Three dimensional microscopy, Confocal Laser Scanning Microscopy (CLSM) provides increased resolution, that can exceed the Raleigh Criteria, and the ability to discretely observe and analyze objects through z space in cells and tissues. Confocal imaging technology can be used to visualize the phenotypic expression of molecular markers or effects of exogenous molecules, drugs, antagonists or agonists on cellular processes.

With the advent of high throughput genomic and proteomic instrumentation for measuring molecular expression, what is the role of confocal imaging technology in drug discovery? The advantage of high resolution three dimensional microscopy is the ability to measure the spatial and temporal distribution of molecules and cellular components which is vital to understanding the activity of drug targets, biomarkers and drug effect at the cellular level (Fig.1). Expression data generated by genomic and proteomic instrumentation on cell free preparations lacks the important spatial component provided by imaging technologies. Three dimensional imaging provides spatial information of expression in $\mathrm{z}$ as well as $\mathrm{x}$ and $\mathrm{y}$ which can be important for the functional expression of molecules [2].

Confocal Microscopy is commonly used in our laboratories in the pre-clinical stages of drug discovery for exploratory studies, target identification, target validation, assay development, and in some instances lead optimization. Confocal Microscopy in the context of generating three/four dimensional images is not a tool for lead generation because of the time required for collecting multiple optical sections. Examples of studies using three dimensional microscopy are: localization, translocation, and co-localization of molecules (Fig 2). Other drug discovery uses are: receptor internalization, cellular interactions, drug interactions with cell components (Fig. 3), and cell signals.

Emerging applications of three dimensional microscopy for drug discovery include: Ex-vivo and intra vital imaging of biological systems with Multiphoton Laser Scanning Microscopy using fluorophores and intrinsic signals such as auto-fluorescence and Second Harmonic signals [2] and andnanometer resolution in optical microscopy [3].

\section{References}

[1] J. Russ, Seeing the Scientific Image, http://drjohnruss.com

[2] B. Blazer-Yost et al, Am J Physiol Cell Physiol (February 26, 2003). 10.1152 
[3] W. Webb et al, PNAS, 100 (12) 20037075

[4] S. Hell et al, Nature BioTechnology 21(11) (2003) 1303
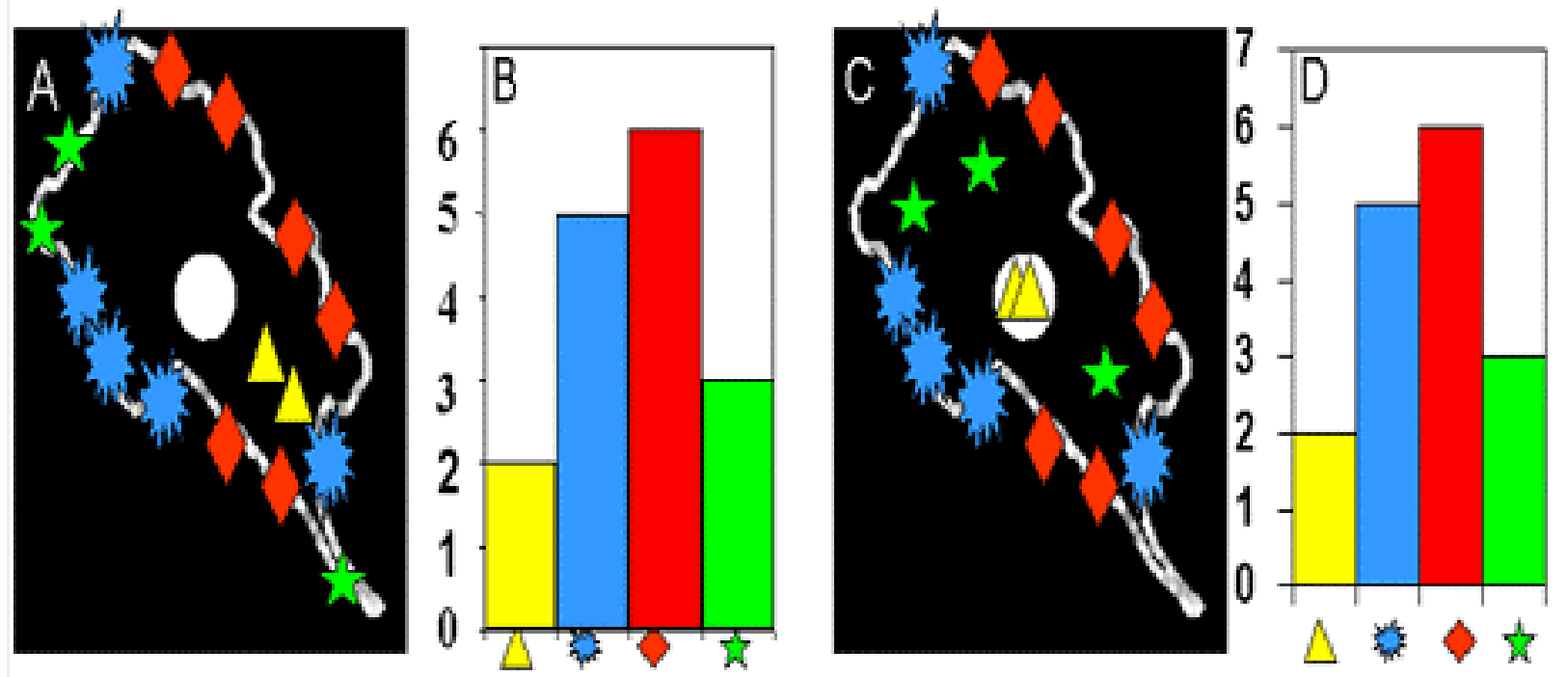

Fig 1. Cell Free Measurements Don't Measure Up. A. Normal Cell spatial distribution of four proteins. B. Cell free measurement of protein or expression of genes in normal cell. C. Diseased cell spatial distribution of proteins. D. Cell free measurement shows no change in quantity but spatial distribution changed.

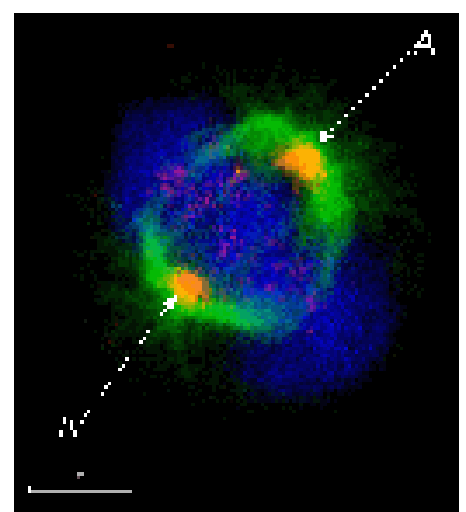

.Fig 2. Co-localization of marker molecule with mitotic spindle pole

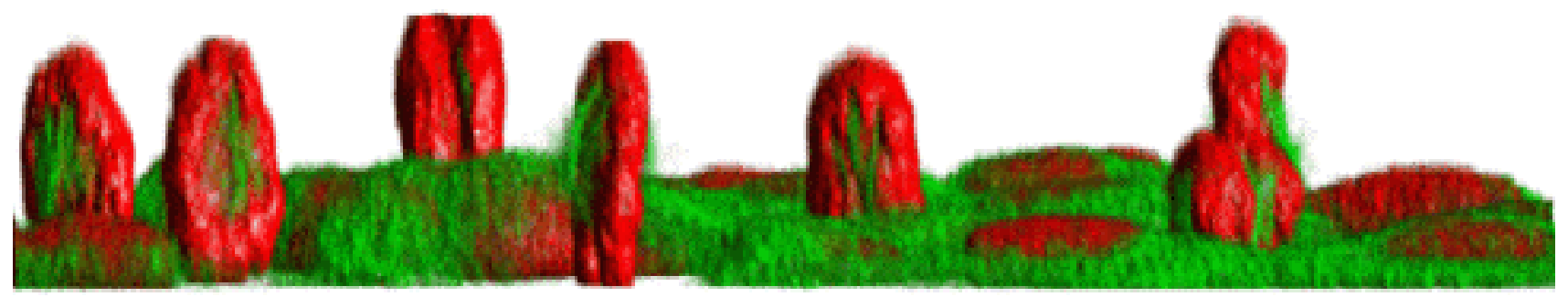

Fig. 3 Effect of drug treatment on mitotic spindle pole separation from the chromatin plate in cancer cells. 